\title{
SINGLE MODE OPERATION OF IMPURITY-INDUCED DISORDERING LARGE AREA VERTICAL CAVITY SURFACE EMITTING LASERS
}

C.W. Lo and S.F. Yu

The University of Hong Kong, Department of Electrical and Electronic Engineering, Pokfulam Road, Hong Kong.

\section{ABSTRACT}

Vertical-cavity surface-emitting lasers (VCSELs) with suitable interdiffusion quantum wells profile by the use of selective impurity-induced disordering is proposed for high power single mode operation in large area devices. It is shown that the transverse optical confinement in the quantum well active region formed by the diffusion profile counteracts the influence of carrier spatial hole burning for VCSELs biased at high injection current. Results indicate that a single mode operation can be maintained in VCSELs with the diameter of core region equal to $50 \mu \mathrm{m}$.

\section{INTRODUCTION}

VCSELs are becoming one of the promising devices for various applications in high bit-rate optical fiber communication system, optical parallel processing and optical interconnections. This is because of their unique features such as small circular output beam divergence, single longitudinal mode operation with low threshold currents less than $1 \mathrm{~mA}$ and high relaxation oscillation frequency [1-3]. However, the excitation of higher order transverse modes degrades VCSELs' performance in high speed optical communication systems and should be avoided. To achieve the above applications, a single fundamental transverse mode operation is essential due to the elimination of noise and instability and the ease of higher coupling efficiency with optical fibers [4]. In index guided VCSELs, the number of modes depends on their transverse dimensions and the refractive index profile between the core and cladding regions. In gain guided VCSELs, the fundamental transverse mode operation can be maintained for large area devices. However, higher order transverse modes are excited with high injection currents due to spatial hole burning effect within the core region [5]. Therefore, it is necessary to suppress the multiple transverse modes in VCSELs at high output power for large area devices.

Concentration of impurity varies the refractive index and carrier diffusion rate of diffused quantum well (DFQW) material [6]. A defined pattern of refractive index profile can be obtained by selective area disordering of quantum well materials and this technique can be utilized for the fabrication of optical devices such as lasers and modulators [7]. In this paper, single mode operation of large area VCSELs using selective disordering of quantum well active region is studied theoretically.

\section{LASER STRUCTURE}

The schematic of VCSEL after selective impurity-induced disordering is shown in figure 1. It is assumed that the active layer consists of $20 \mathrm{Al}_{0.3} \mathrm{Ga}_{0.7} \mathrm{As} / \mathrm{GaAs}$ quantum wells with well width and barrier thickness of $100 \AA$ and $100 \AA$, respectively. In order to achieve a single mode operation of VCSELs, the transverse optical confinement in the quantum well active region is produced by the use of selective impurity-induced disordering technique. The selective injection of impurities can be done by using a circular mask located at the center of the core region to 
shelter from the ion implantation. A suitable distribution of impurity along the transverse direction of impurity can be obtained with appropriated annealing time and temperature. Therefore, dimension of mask, implantation energy, annealing temperature and time are the parameters to optimized the gain margin of VCSELs.

\section{LASER MODEL}

The multimode rate equation model of VCSEL used in our analysis are given as follows [8]

$$
\begin{gathered}
\frac{\partial N(r, t)}{\partial t}=\frac{J(r, t)}{\mathrm{qd}_{\mathrm{k}}}-\frac{\mathrm{N}(\mathrm{r}, \mathrm{t})}{\tau}+\mathrm{D} \frac{1}{\mathrm{r}} \frac{\partial}{\partial \mathrm{r}}\left(\mathrm{r} \frac{\partial \mathrm{N}(\mathrm{r}, \mathrm{t})}{\partial \mathrm{r}}\right)-v_{\mathrm{g}} \Gamma_{\mathrm{z}} \mathrm{G}(\mathrm{r}, \mathrm{t}) \sum_{\mathrm{i}}\left|\mathrm{E}_{\mathrm{i}}(\mathrm{r})\right|^{2} \mathrm{~S}_{\mathrm{i}}(\mathrm{t}) \\
\frac{\partial \mathrm{S}_{\mathrm{i}}(\mathrm{t})}{\partial \mathrm{t}}=v_{\mathrm{g}}\left(\Gamma_{\mathrm{z}} \mathrm{G}_{\mathrm{i}}-\alpha_{\mathrm{i}}\right) \mathrm{S}_{\mathrm{i}}(\mathrm{t})+\mathrm{R}_{\mathrm{sp}}(\mathrm{t})
\end{gathered}
$$

where $\mathrm{N}$ is the transverse distribution in the radial direction, $\mathrm{r}$ of carrier concentration inside the active layer and $S_{i}$ is photon density of the ith mode. $d_{k}$ is the thickness of the active layer, $q$ is the electron charge, $\tau$ is the carrier lifetime, $\Gamma_{2}$ is the longitudinal confinement factor, $v_{\mathrm{g}}\left(=\mathrm{c} / \mathrm{n}_{\mathrm{z}}\right.$, where $\mathrm{n}_{\mathrm{g}}$ is the group index and $\mathrm{c}$ is the velocity of light in free space) is the group velocity and $\mathrm{D}$ is the ambipolar diffusion coefficient. $\alpha_{i}$ is the scattering loss in the active layer for ith mode and Rsp is the spontaneous emission rate. $J(r, t)$ is the current density distribution along the transverse direction.

In the photon rate equation, the effective optical gain $\mathrm{G}_{\mathrm{i}}$ of the ith mode is given by

$$
\mathrm{G}_{\mathrm{i}}=\frac{2}{\mathrm{w}^{2}} \int_{0}^{\infty} \mathrm{g}(\mathrm{N}) \mid \mathrm{E}_{\mathrm{i}}(\mathrm{r}, \mathrm{t})^{2} \mathrm{rdr},
$$

where $g$ is the optical gain of the quantum well's active layer to be determined

The symbol $E_{i}$ represents the normalized slowly varying complex field intensity (i.e. $\left.\mathrm{l}=2 \int_{0}^{\infty}\left|\mathrm{E}_{\mathrm{i}}\right|^{2} \mathrm{rdr} / \mathrm{w}^{2}\right)$ for the ith mode with $\mathrm{S}_{1}$ as the corresponding photon density. The field intensity, $\mathrm{E}_{\mathrm{i}}$, can be determined by solving the complex scalar Helmholtz equation. It is expected that the active layer supports the transverse modes which can be expressed in terms of linearly-polarized $\left(\mathrm{LP}_{\mathrm{p}}\right)$ modes where the indexes $i$ and $\mathrm{p}$ denote the azimuthal and radial order of modes respectively. The $\mathrm{LP}_{01}$ mode is dominated due to its maximized overlapping with the optical gain profile, however, the $L P_{4}$ mode can also be excited for the reasons of non-uniform current injection and spatial hole burning effects. Therefore, the influence of modes competition (between $\mathrm{LP}_{01}$ and $\mathrm{LP}_{11}$ modes) is also taken into calculation. The subscript $\mathrm{i}=1$ and $\mathrm{i}=2$ of the above equations represent the case of $L P_{01}$ and $L P_{11}$ mode respectively.

The threshold and above threshold behavior of VCSELs with selective disordering is obtained from equation (1) and (2) by setting the time-derivatives equal to zero. These non-linear and time independent simultaneous rate equations can be solved in a self-consistent manner. The lateral variation along carrier concentration of the active layer is also solved by using finite difference method subject to the condition that $\mathrm{N}$ and its derivative are continuous everywhere. If we assume the circular symmetry of carrier concentration, at $r=0$, the first and second derivative of $\mathrm{N}$ take the form as follow :

$$
\begin{gathered}
\left.\frac{\partial N}{\partial r}\right|_{r_{1}}=0 \\
\left.\frac{\partial^{2} N}{\partial r^{2}}\right|_{r_{1}}=\frac{2\left(N\left(r_{2}, t\right)-N\left(r_{1}, t\right)\right)}{\Delta r^{2}}
\end{gathered}
$$




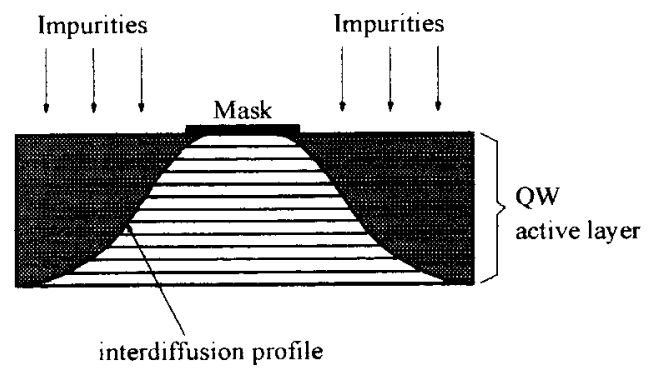

Figure 1. Schematic of a VCSEL after selective disordering
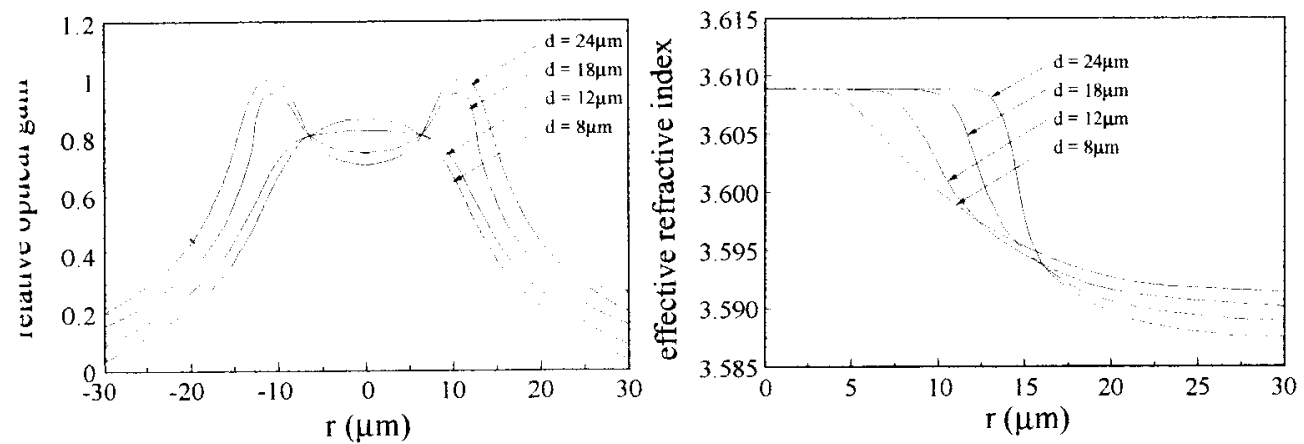

Figure 2. The optical gain and refractive index distribution of VCSEL with the diameter of mask, $\mathrm{d}$ varied between 8 to $24 \mu \mathrm{m}$.

where $\Delta r$ is the separation between two successive points in the radial direction, $r$. It is also required that as $r \rightarrow$ very large, the carrier concentration change $N \rightarrow 0$. The field intensity $E_{i}$ is also solved simultaneously with the carrier concentration such that the time-dependent transverse field intensity variation can also be considered into investigation.

\section{MODEL FOR QUANTUM WELL MATERIAL}

The refractive index and optical gain of quantum wells material under the influence of impurities induced disordering are also considered in our analysis. The models given in references $[9,10,11]$ are utilized to calculate the optical and electrical properties of DFQW's which are summarized in Appendix A. It is defined that the extent of interdiffusion into the quantum wells material is characterized by a diffusion length, $L_{d}$, where $L_{d}=0 \AA$ represents the asgrown quantum wells and the diffusion strength of impurity is described by the magnitude of $L_{d}$. The corresponding magnitude of $L_{d}$ between the mask and non-mask region are equal to 0 and $10 \AA$, respectively. In the following calculation, the values of DFQW's parameters vary with $L_{d}$ and device parameters used in the laser model are given in Table I and II, respectively. 
Table 1. Material Parameters in Laser Structure

\begin{tabular}{|c|c|c|}
\multirow{2}{*}{$\begin{array}{c}\text { (at operating wavelength } \\
\text { of } 0.85 \mu \mathrm{m})\end{array}$} & \multicolumn{2}{c|}{ Diffusion Length $\left(\mathrm{L}_{\mathrm{d}}\right)$} \\
\cline { 2 - 3 } & $0 \AA$ & $10 \AA$ \\
\hline Fitted parameters $\left(\mathrm{a}_{\mathrm{N}}\right) \mathrm{cm}^{-1}$ & 1780.0121 & 468.6990 \\
\hline $\begin{array}{c}\text { Transparency carrier density } \\
\left(\mathrm{N}_{\mathrm{o}}\right) \times 10^{18} \mathrm{~cm}^{-3}\end{array}$ & 2.1996 & 2.4092 \\
\hline Fitted parameters $(\mathrm{e})$ & -0.02824 & -0.02521 \\
\hline Fitted parameters $\left(\mathrm{N}_{\mathrm{r}}\right) \times 10^{18} \mathrm{~cm}^{-3}$ & 2.0673 & 1.9809 \\
\hline Refractive index $\left(\mathrm{n}_{\mathrm{B}}\right)$ & 3.627 & 3.5880 \\
\hline
\end{tabular}

Table II. Parameters Used in Model

\begin{tabular}{|c|c|}
\hline Parameters (symbol) & Magnitude \\
\hline Thickness of active region $(d)$ & $1 \mu \mathrm{m}$ \\
\hline Carrier lifetime $(\tau)$ & $4 \mathrm{~ns}$ \\
\hline Effective group refractive index $\left(\mathrm{n}_{\mathrm{L}}\right)$ & 3.70 \\
\hline Longitudinal confinement factor $\left(\Gamma_{z}\right)$ & 0.16 \\
\hline Velocity of light in free space $(\mathrm{c})$ & $3 \times 10^{10} \mathrm{cms}^{-1}$ \\
\hline Ambipolar diffusion coefficient $(\mathrm{D})$ & $15 \mathrm{~cm}^{2} \mathrm{~s}^{-1}$ \\
\hline
\end{tabular}

\section{RESULTS \& DISCUSSIONS}

It is assumed that the laser is initially biased at threshold and modulated by a step current of 2 times its threshold value. The operating wavelength of the VCSEL is chosen to be $\lambda_{0}=0.85 \mu \mathrm{m}$ and the diameter of core region is equal to $50 \mu \mathrm{m}$. The diameter of circular mask, $\mathrm{d}$ is varied between 8 and $24 \mu \mathrm{m}$ to shelter from the ion implantation. In order to ensure the current is effectively injected in the laser, the current confinement structure is needed. It is observed that the relative optical gain at the center of core region of DFQW-VCSEL is suppressed for the increasing diameter of circular mask. With the cases of the diameter of circular mask equal to $8 \mu \mathrm{m}$ and $12 \mu \mathrm{m}$, the transverse optical confinement in the quantum well active region is produced. This is because a gaussian distribution of permittivity along the active layer is produced by the impurities andthe transverse field is guided. The gain margin can also be enhanced if the overlapping integral between the optical gain and the fundamental transverse mode is maximized.

Figure 3 shows the near field profile of DFQW-VCSEL with the diameter of circular mask varied between 8 and $24 \mu \mathrm{m}$. It is found that the fundamental mode of optical field can not be maintained for large size of circular mask. This is because the active layer of DFQW-VCSEL with large circular mask no longer supports the $L_{0}$ transverse mode. Moreover, the excitation of higher-order transverse mode is observed due to the spatial hole burning effects. Therefore, the dimension of the circular mask can affect the performance of DFQW-VCSEL. 

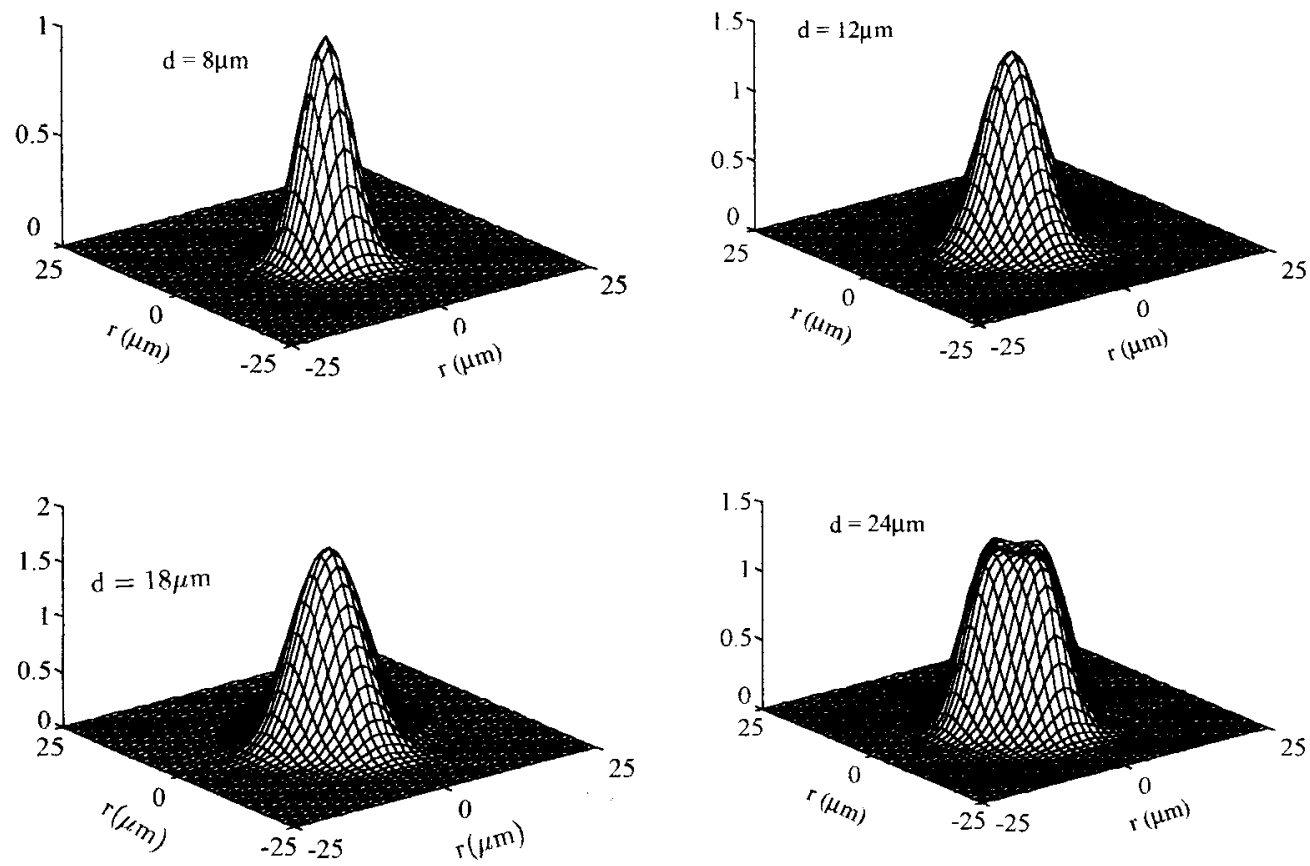

Figure 3. The near field profile with the diameter of mask, d, varied between 8 to $24 \mu \mathrm{m}$.

\section{CONCULSION}

In conclusion, the selective impurity-induced disordering vertical-cavity surface-emitting lasers with the diameter of the core region equal to $50 \mu \mathrm{m}$ is investigated. These devices are designed to achieve a high power single mode opearion at $0.85 \mu \mathrm{m}$. Results show that the single mode operation with the diameters of the circular mask varied between 8 and $18 \mu \mathrm{m}$ are obtained. The confinement of the transverse optical field is produced by using selective IID technique can reduce the influence of the carrier spatial hole burning at high injection current. Therefore, the behaviour of the DFQW-VCSEL is affected by the size of the circular mask. Besides, the implanation energy, annealing temperature and time are also the important parameters to determine the modal behaviour of DFQW-VCSEL.

\section{APPENDIX A}

The refractive index, $\mathrm{n}_{\mathrm{DFQW}}$, of diffused quantum wells active layer is given by [9]

$$
\mathrm{n}_{\mathrm{DFQW}}(\omega)=\left(\frac{1}{2} \varepsilon_{1}^{\mathrm{T}}(\omega)+\frac{1}{2}\left\{\left[\varepsilon_{1}^{\mathrm{T}}(\omega)\right]^{2}+\left[\varepsilon_{2}^{\Gamma}(\omega)\right]^{2}\right\}^{1 / 2}\right)^{1 / 2}
$$

where $\omega$ is the angular frequency, $\varepsilon_{1}^{\Gamma}(\omega)$ and $\varepsilon_{2}^{\Gamma}(\omega)$ are the real and imaginary parts of the total dielectric function for the $\Gamma$ valley, respectively. 
Using density matrix approach, the optical gain with photon generated in the direction perpendicular to the surface of quantum well layers is given as [11]

$$
\begin{aligned}
\mathrm{g}(\omega) & =\frac{\mathrm{e}^{2} \mathrm{M}_{\mathrm{b}}^{2}}{\pi \mathrm{c} \varepsilon \mathrm{m}_{\mathrm{o}}^{2} \omega L_{z}} \sum_{\mathrm{p} . \mathrm{q}} \int\left\langle\left.\left\langle\psi_{\mathrm{c}_{\mathrm{p}}} \mid \psi_{\mathrm{vq}_{\mathrm{q}}}\right\rangle\right|^{2} \mathbf{P}_{\mathrm{pq}}(\mathrm{k})\right. \\
& \times \mathrm{L}\left[\mathrm{E}_{\mathrm{p}}(\mathrm{k})-\mathrm{E}_{\mathrm{q}}(\mathrm{k})-\eta \omega\right)\left\{\mathrm{f}^{\mathrm{c}}\left[\mathrm{E}_{\mathrm{p}}(\mathrm{k})\right]-f^{v}\left[E_{q}(k)\right]\right\} \mathrm{dk}
\end{aligned}
$$

where $m_{0}$ is the rest mass of electron and $M_{b}$ is the optical matrix. $E_{p}$ and $E_{q}$ are the pth-electron and qth-hole sub-band-edge energy, respectively, $\psi_{\mathrm{C}}$ and $\psi_{\mathrm{V}}$ are the envelope wavefunctions for the electrons and holes, respectively. $L$ is the Lorentzian broadening factor with HWHM of $5 \mathrm{meV}$ and $\mathrm{k}$ is the wavevector. The summation in (A2) is over all the conduction and valence sub-bands and $\mathbf{P}(\mathbf{k})$ is the TE-polarization factor. $\mathrm{f}^{\mathrm{C}}$ and $\mathrm{f}^{\mathrm{V}}$ are the quasi-Fermi for the electrons in the conduction and valance bands, respectively.

It is noted from our calculation that at a particular $L_{d}$ and at an external carrier injection level, $\mathrm{N}$, the TE net optical gain spectrum at room temperature is found to have a simple expression, $g(\omega)=a_{N} \ln \left(N / N_{0}\right)$, where $a_{N}$ is the fitted parameter and $N_{0}$ is the carrier concentration at transparency. The parameters $a_{N}$ and $N_{0}$ at particular frequency vary with $L_{d}$ are given in Table 1 .

The carrier induces refractive index change, $\Delta \mathrm{n}$, which varies with the background refractive index profile of active region, can be obtained from the change of gain coefficient, $\Delta \mathrm{g}(\omega)=\mathrm{g}(\omega)-\mathrm{g}_{0}(\omega)$, through the Kramers-Kronig dispersion relation [10]

$$
\Delta \mathrm{n}(\omega)=\frac{\pi}{\mathrm{c}} \mathrm{PV} \int_{0}^{\infty} \frac{\Delta \mathrm{G}\left(\omega^{\prime}\right)}{\omega^{\prime 2}-\omega^{2}} \mathrm{~d} \omega^{\prime}
$$

where $g_{0}(\omega)$ is the optical gain at transparency. The symbol PV stands for the Cauchy principle value. We can also show that at a particular $L_{d}$ the relation between $\Delta n$ and $N$ can be expressed as, $\Delta \mathrm{n}=\mathrm{e} \ln \left(\mathrm{N} / \mathrm{N}_{\mathrm{r}}\right)$, where e and $\mathrm{N}_{\mathrm{r}}$ are fitted parameters vary with $\mathrm{L}_{\mathrm{d}}$ and also given in Table $1 \mathrm{l}$.

\section{REFERENCES}

[1] Y. H. Lee, B. Tell, K. Brown-Goebeler, J. L. Jewell and J. V. Hove, Electron. Lett., 26, 710 (1990).

[2] R. S. Geels and L. A. Coldren, Appl. Phys. Lett., 57, 1605 (1990).

[3] D. Tauber, G. Wang, R. S. Geels, J. E. Bowers, and L. A. Coldern, Appl. Phys. Lett, 62, 325 (1993).

[4] C. J. Chang-Hasnain, M. Orenstein, A. Von Lehmen, L. T. Florez, J. P. Harbison and N.G. Stoffel, Appl. Phys. Lett., 57, 218 (1990).

[5] A. Valle, J. Sarma and K. A. Shore, IEEE J. Quantum Electron, 31, 1423 (1995).

[6] E. H. Li and B. L. Weiss, IEEE J. Quantum Electron., 29, 311 (1993).

[7] R. P. Bryan, J. J. Coleman, L. M. Miller, M. E. Givens, R. S. Averback and J. L. Klatt, Appl Phys. Lett., 55, 94 (1989).

[8] G. Ronald Hadley, K. L. Lear, M. E. Warren, K. D. Choquette, J. W. Scott and S. W. Corzine, IEEE J. Quantum Electron., 32, 607 (1996).

[9] E.H. Li, B.L. Weiss, K.S. Chan and J. Micallef, Appl. Phys. Lett. 62, 550, (1992).

[10] C.H. Herny, R.A. Logan and K.A. Bertness, J. Appl. Phys. 52, 4457, (1981).

[11] E.H. Li and K.S. Chan, Electron. Lett., 29, 1233, (1993) 